\title{
Los efectos en las políticas públicas del gobierno de la Cuarta Transformación en la educación superior en México
}

\author{
Elia MARÚm ESPINOSA* \\ Carmen EnEdina Rodríguez Armenta**
}

*Directora y Profesora Investigadora del Centro para la Calidad e Innovación de la Educación Superior del Centro Universitario de Ciencias Económico-Administrativas (CUCEA) de la Universidad de Guadalajara (U de G).

**Directora de Educación Superior Universitaria de la Subsecretaría de Educación Superior de la Secretaría de Educación Pública y Profesora Investigadora del CUCEA de la U de G.

\section{Introducción}

La realidad que estamos viviendo enfrenta vertiginosas transformaciones en el entorno local, nacional y mundial, como característica de la tercera década del presente siglo, marcada por cambios e innovaciones disruptivas, pero también por inercias y continuidades. La educación superior ha devenido como elemento estratégico para el desarrollo sostenible de las naciones, y por ello protagonista y receptora de estas transformaciones donde las políticas públicas y las formas de gestión cambian y generan efectos nuevos de diverso tipo, de ahí la necesidad de contribuir con perspectivas académicas que nos permitan conocer y valorar algunos de estos efectos, finalidad fundamental del presente artículo.

El gobierno de México inicia este 2020 no solo inmerso en las transformaciones tecnológicas y los impactos de la llamada Cuarta Revolución Industrial o Revolución 4.0, sino con un nuevo proyecto de nación y un nuevo enfoque de gobierno. Dicho cambio se asemeja a las tres transformaciones surgidas de los grandes movimientos sociales del siglo XIX y XX: la Independencia nacional; la Guerra de Reforma; y la Revolución Mexicana. El actual gobierno impulsa la Cuarta Transformación, guiada por políticas públicas de austeridad en el gasto público, mesura en el tamaño y funcionamiento del aparato gubernamental, los pobres como centro y objetivo central de las políticas públicas, transparencia y cero tolerancia a la corrupción en la gestión pública, y en la educación, la elevación a rango constitucional de la educación superior como derecho humano, y por tanto, su universalización y gratuidad en todos sus niveles. 
El gobierno federal, al inicio del presente año, se ve interpelado por la pandemia del coronavirus denominada Covid-19 que ha impactado, aún sin fecha clara de terminación, sus planes y proyectos y su propio funcionamiento, rompiendo el paradigma vigente y desafiando su flexibilidad para cambiar y su capacidad para hacerlo con gran rapidez. El sector educativo y el subsector de educación superior, inmersos en este desafío se han encontrado entre dos lógicas de actuación y entre dos tipos de políticas públicas; las diseñadas y aplicadas al inicio de la actual administración del gobierno federal, y las emergentes ante la pandemia, en un ambiente marcado desde el inicio del sexenio, por cierta desconfianza hacia la actuación, respuestas y resultado social del trabajo de las universidades, lo que se ha reflejado sobre todo, en las políticas presupuestales, la ampliación de la oferta educativa a través de las propias capacidades institucionales de las universidades autónomas y la creación del Sistema de Universidades para el Bienestar Benito Juárez, modelo operado de manera centralizada y con la expectativa de tener 100 universidades nuevas en el país.

\section{Trayectorias desde el 2015 de las políticas de apoyo a la educación superior en México}

El Centro Interuniversitario de Desarrollo (CINDA), con sede en Chile, prepara cada cinco años un diagnóstico amplio de la educación superior en Iberoamérica (Brunner y Miranda, 2016), su informe más reciente corresponde al periodo 2010-2015, y consta de un panorama general y un informe para cada país. En el Informe País, México (Marúm, 2016) se concluye que el subsistema de educación superior mexicano no presentó grandes cambios durante el quinquenio estudiado, pero los cambios que enfrentó fueron de importancia en su consolidación y avance al tener un aumento, aunque moderado, en la matrícula de pregrado, siendo una de las coberturas más bajas de América Latina y de la OCDE, y un aumento, moderado también, de la matrícula en posgrado y de las becas para cursar posgrados de calidad. Esta situación no ha cambiado mucho hasta la actualidad con el nuevo régimen de gobierno, lo que sí cambió fue la tendencia para crear nuevas universidades y nuevos criterios para reconocer y apoyar la investigación y los posgrados.

En la actualidad continúa la tendencia ya detectada, en el informe mencionado, del incremento del índice inflacionario del presupuesto y los programas financieros gubernamentales -aunque insuficientes- para ampliar la matrícula y los esfuerzos institucionales para lograrlo, lo cual ha abierto la puerta a miles de jóvenes que no tenían esta opción, dichos programas continúan y se fortalecen a pesar del complejo panorama financiero mundial. La ampliación del programa de becas de manutención y apoyos a estudiantes; la inclusión de la perspectiva de género en los planes gubernamentales e institucionales y los apoyos para mujeres y estudiantes en condiciones especiales, particularmente para quienes tienen alguna discapacidad o quienes pertenecen a pueblos y comunidades indígenas, continúan y se retoman, donde se han incluido a los pueblos y grupos interculturales y los afromexicanos debido a su gran importancia cultural. 
Una de las características de las políticas públicas más importantes ha sido legitimar la evaluación y la acreditación, buscando avanzar hacia esquemas internacionales que justifiquen las políticas públicas para la calidad educativa, así como el aumento moderado al apoyo a docentes ante los requisitos de las políticas públicas de calidad. El crecimiento de investigadores en la Academia, en especial quienes gocen del más alto nivel como miembros del Sistema Nacional de Investigadores (SNI); el aumento de programas de apoyo y financiamiento a la $I+D+i$ (Investigación más Desarrollo más Innovación Tecnológica); el inicio de la búsqueda de una normatividad propia para la educación superior y la estabilidad del subsistema de educación superior que le ha permitido trabajar sin paros de labores ni enfrentamientos, son características de las políticas públicas de la segunda década del presente siglo, señala el informe del CINDA. Sin embargo, existen problemas $\mathrm{y}$ asuntos que se hallaban en discusión desde 2015 y que todavía prevalecen en la actualidad. De acuerdo con el Informe País (CINDA, 2016) estos son:

Ampliar la cobertura más allá de $40 \%$ de tasa neta (no de tasa bruta como se ha medido) e incluso ampliarla a $60 \%$ para tener posibilidades de sustentabilidad de nuestro desarrollo; realizar las reformas jurídicas para contar con un modelo de financiamiento de la educación superior con proyección plurianual y asegurar que se destine un porcentaje del PIB a este nivel educativo y a la $I+D+i$, acorde con las necesidades del desarrollo sustentable de México; ampliar la planta docente e incentivar una generación de reemplazo, sustituyendo las plazas de jubilados, reconociendo la definitividad de docentes bajo contrato e incorporando jóvenes talentos; ampliar, agilizar, articular y armonizar el Sistema de Información para hacerlo accesible y útil a todas las necesidades del subsistema de educación superior; encontrar e impulsar un nuevo modelo de acreditación que no esté ya basado en insumos y en indicadores de primera generación, sino que se fundamente en resultados y en indicadores de tercera generación; reglamentar la acreditación internacional y a las agencias y organismos acreditadores; Incluir en las discusiones y análisis al 
Tratado de Libre Comercio entre México, Estados Unidos y Canadá (T-MEC) y los impactos que traerá para el subsistema; incluir la perspectiva de género no solo en el presupuesto, sino en las políticas y práctica cotidiana de las Instituciones de Educación Superior (IES) y del gobierno; diseñar una política para aprovechar los escasos cuatro años que nos quedan del bono demográfico, bono que se agota en 2020, y armonizar actuarialmente la ampliación de la oferta a la dinámica y estructura poblacional; enfrentar con políticas imaginativas y comprometidas socialmente la subutilización de la infraestructura educativa no solo del subsistema de educación superior sino de todo el sistema educativo para apoyar con más fuerza el aumento de la matrícula; retomar el derecho a la educación a lo largo de la vida y seguir impulsando programas para adultos mayores y universidades para estos adultos, así como los programas de la universidad incluyente para indígenas y discapacitados y para jóvenes de escasos recursos económicos; integrar los Objetivos del Desarrollo Sustentable (ODS) a la educación superior y reconocer a la educación superior como un elemento estratégico para el logro del desarrollo sustentable; pero sobre todo y primero que todo, avanzar en la construcción de un verdadero sistema nacional de información armonizado, que tenga accesibilidad y disponibilidad de la información hasta los niveles más desagregados permitidos por la ley (Marúm, 2016).

Como puede observarse en esta larga cita, los pendientes desde hace un quinquenio prevalecen y se constituyen en uno de los retos a enfrentar por las políticas públicas del actual gobierno federal, pero los mayores retos son: transformar la educación lineal fabril estandarizada para una economía lineal basada en la ganancia y en el progreso material a toda costa, por una educación integral e innovadora, basada en el aprendizaje vivencial y activo, que incluya la interculturalidad, la cultura de paz y la educación en la comunidad para la nueva economía social y solidaria, con una gestión del conocimiento y no fabril estandarizada, y asegurar la universalización y gratuidad de este nivel educativo, en un entorno restrictivo para la economía y para los ingresos del gobierno.

\section{El marco contextual y legal de la educación superior en el nuevo gobierno mexicano}

El final de la segunda década del presente siglo marca un parteaguas en algunas de las políticas públicas para la educación superior, así como la continuidad e inercia en otras. El arribo al gobierno federal de un candidato de oposición, con un modelo de gobernanza con fuertes diferencias de los anteriores regímenes, pero también con muchas similitudes, inicia, desde una perspectiva distinta, la revisión del sistema de educación en México, pero en especial de la Educación Superior, subsistema que no contaba con una normatividad completa y actualizada, y al menos se tiene la intención al existir un anteproyecto de Ley General de Educación Superior, ley a la que en otros países como España se complementa con una Ley de Autonomía Universitaria, aún no planteada para México, donde la autonomía es referida solo a las fracciones correspondientes del Artículo Tercero Constitucional. 


\section{EL POLVO DE LOS CAMINOS}

Por Abel Quezada

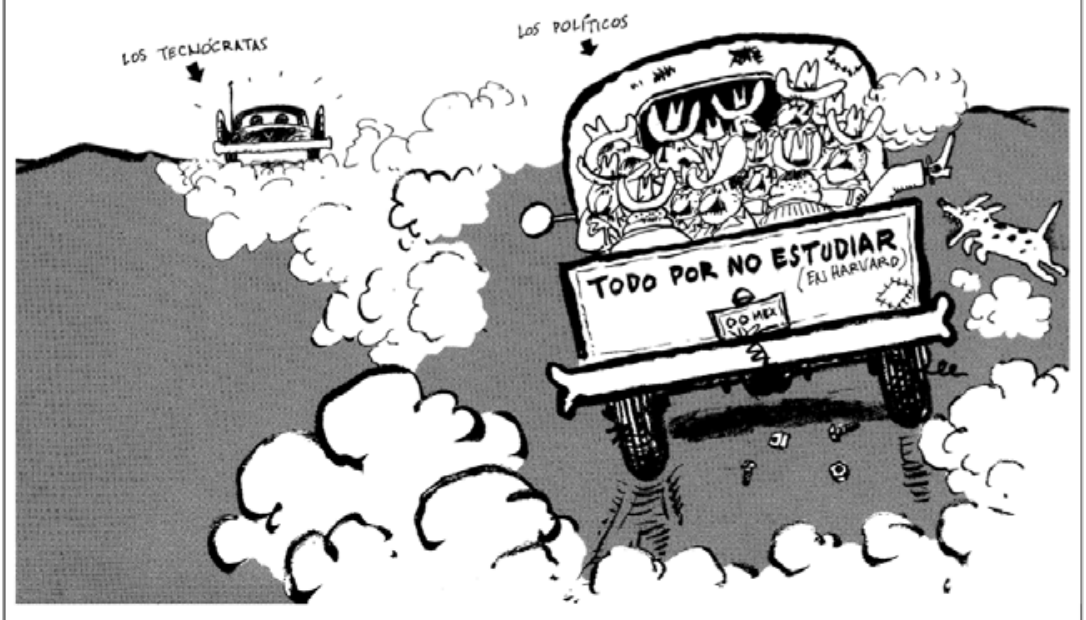

Novedades. Número 14,666. Sección editorial. Página 5. Martes 3 de noviembre, 1981.

La anterior Ley General de Educación de 1993 especificaba que la educación que ofrezca el Estado "será de calidad, entendiéndose por ésta la congruencia entre los objetivos, resultados y procesos del sistema educativo, conforme a las dimensiones de eficacia, eficiencia, pertinencia y equidad" siendo un ejemplo de conceptualización propia, en sus dos primeros elementos, del enfoque de la gestión pública del New Public Management, inspirado en los principios empresariales de la gestión.

El nuevo modelo para la gobernanza y gestión del Sistema Educativo Mexicano actual se plasmó en las reformas constitucionales de 2019, a los Artículos 3, 31 y 73 Constitucionales. El artículo $3^{\circ}$ de la Constitución Política de los Estados Unidos Mexicanos establece que la educación deberá ser:

[...] de excelencia, entendida ésta como el mejoramiento integral constante que promueve el máximo logro de aprendizaje de los educandos, para el desarrollo de su pensamiento crítico y el fortalecimiento de los lazos entre escuela y comunidad debiendo considerarse la pertinencia y la relevancia, con lo que se presenta un cambio en el marco normativo y en el enfoque para las políticas públicas, alejándose del enfoque empresarial y determinando a la educación superior como un derecho humano. Elemento fundamental en los nuevos enfoques de política pública, donde la educación superior se considera obligatoria y gratuita por parte del Estado mexicano, y donde se deberá cumplir a cabalidad otorgando los recursos suficientes y necesarios para otorgarla a lo largo y ancho del territorio nacional. 


\section{Diversidad y complejidad del subsistema de educación superior mexicano}

La diversidad de la naturaleza y organización de las instituciones de educación superior que forman este subsector en México tiene como referente las recomendaciones de organismos supranacionales como el Banco Mundial y la Organización para la Cooperación y Desarrollo Económicos (OCDE) que desde los años 90 promovieron la creación de universidades alternativas a las tradicionales universidades federales y estatales y al reducido sistema tecnológico, por lo que en los siguientes años se fueron creando diversas instituciones sobre todo tecnológicas. Así el subsector de educación superior mexicano está conformado por 945 instituciones: Universidades Públicas Federales; Universidades Públicas Estatales, Universidades Tecnológicas; Universidades Públicas Estatales con Apoyo Solidario; Institutos Tecnológicos, Universidades Politécnicas; Universidad Pedagógica Nacional; Universidad Abierta y a Distancia, Universidades Interculturales; Escuelas Normales Públicas; Centros Públicos de Investigación; otras Instituciones Públicas entre las que se encuentran las creadas por el actual gobierno federal; las Universidades para el Bienestar Benito Juárez García.

Si bien esta cantidad es grande, la complejidad es mayor al existir gran número de instituciones de naturaleza similar o complementaria, pero que no conforman un verdadero subsistema, sino que cada una, según su naturaleza y funciones, actúan de manera atomizada o poco coordinada, de ahí que las actuales políticas públicas busquen la articulación de ellas para crear un verdadero sistema cuyos esfuerzos sean concatenados y dirigidos a los mismos objetivos. El Subsecretario de Educación Superior (Concheiro, 2020) reconoció que la educación superior es un archipiélago de archipiélagos y no un subsistema dentro de un sistema de educación superior, el cual tampoco existe articulado y vertebrado entre niveles y sistemas.

En la siguiente tabla se presenta la conformación numérica del tipo de instituciones de este subsistema. 
Total de instituciones de Educación Superior (México, 2019)

\begin{tabular}{|l|c|}
\hline \multicolumn{1}{|c|}{ Universidades e Institutos Públicos } & Número \\
\hline Universidades Públicas Federales & $11^{*}$ \\
\hline Universidades Públicas Estatales & 35 \\
\hline Universidades Tecnológicas & 104 \\
\hline Universidades Públicas Estatales con apoyo solidario & 23 \\
\hline Centros de Investigación (Inst. Tecnológicos) & 6 \\
\hline Institutos Tecnológicos Federales & 126 \\
\hline Institutos Tecnológicos Descentralizados & 130 \\
\hline Universidades Politécnicas & 51 \\
\hline Universidades Interculturales & 10 \\
\hline Subtotal 1 & 494 \\
\hline
\end{tabular}

\begin{tabular}{|l|c|}
\hline \multicolumn{1}{|c|}{ Escuelas Normales y Centros de formación Magisterial } & \\
\hline Aguascalientes & 5 \\
\hline Baja California & 12 \\
\hline Baja California Sur & 3 \\
\hline Campeche & 11 \\
\hline Chiapas & 19 \\
\hline Chihuahua & 4 \\
\hline Coahuila & 8 \\
\hline Colima & 2 \\
\hline Ciudad de México & 6 \\
\hline Durango & 6 \\
\hline Guanajuato & 4 \\
\hline Guerrero & 12 \\
\hline Hidalgo & 7 \\
\hline Jalisco & 11 \\
\hline México & 42 \\
\hline Michoacán & 8 \\
\hline Morelos & 3 \\
\hline Nayarit & 3 \\
\hline Nuevo León & 3 \\
\hline Oaxaca & 14 \\
\hline Puebla & 10 \\
\hline Querétaro & 3 \\
\hline Quintana Roo & 3 \\
\hline San Luis Potosí & 6 \\
\hline Sinaloa & 6 \\
\hline Sonora & \\
\hline & 2 \\
\hline
\end{tabular}




\begin{tabular}{|l|c|}
\hline Tabasco & 6 \\
\hline Tamaulipas & 11 \\
\hline Tlaxcala & 8 \\
\hline Veracruz & 8 \\
\hline Yucatán & 6 \\
\hline Zacatecas & 5 \\
\hline Subtotal 2 & 264 \\
\hline Número de IESP (Subtotal 1+Subtotal 2) & 758 \\
\hline Universidades para el Bienestar Benito Juárez García** & 100 \\
\hline Otras Instituciones de Educación Superior Públicas & 87 \\
\hline Número total de Instituciones de Educación Superior Privadas (IESP) & 945 \\
\hline
\end{tabular}

* Incluye a la Universidad Pedagógica Nacional y a la Universidad Abierta y a Distancia de México creada el 20 de enero de 2012 mediante decreto presidencial.

** Algunas están en proceso de iniciar su funcionamiento.

Fuentes: https://educacionsuperior.sep.gob.mx y https://educacionsuperior.sep.gob.mx/ otras_ies.html

En esta conformación inferimos que la creación de instituciones en el área tecnológica y la creación de escuelas normales, ha respondido a dinámicas políticas y necesidades propias de las localidades y regiones, pero que no ha habido una política pública, ni una respuesta estructurada con un objetivo y una estrategia nacional bien definida, derivada del proyecto de nación en los años previos, dirigida a construir un subsistema, situación que las actuales políticas públicas han planteado y han plasmado su intención de solución tanto en el Proyecto de la Ley General de Educación Superior como en el Programa Sectorial de Educación 2020-2024.

\section{Hacia una Ley General de Educación Superior}

Se está promoviendo la aprobación de la Ley General de Educación Superior, como se señaló, que será marco normativo de las políticas estatales. Este proyecto de ley reconoce que la educación superior es un derecho humano y un bien público social, que será garantizado por el Estado, en los términos del artículo $3^{\circ}$ de la Constitución Política de los Estados Unidos Mexicanos y la impartida por el Estado será universal, inclusiva, pública, gratuita y laica y que las instituciones de educación superior establecerán los respectivos requisitos de admisión, permanencia y titulación, así como las medidas pertinentes para fomentar la inclusión, continuidad y egreso oportuno y que en todo momento se respetará el carácter de las universidades e instituciones a las que la ley les otorga autonomía, la cual se reconoce con base en lo establecido en la fracción VII del Artículo $3^{\circ}$ Constitucional.

Un aspecto importante de este proyecto de ley establece, por primera vez, la relación entre la educación superior y la ciencia, la tecnología y la innovación al señalar que a fin de articular el Sistema Nacional de Educación Superior con el Sistema Nacional de Ciencia y Tecnología e Innovación, las políticas de educación superior y las destinadas a ciencia, humanidades, 
tecnología e innovación, buscarán la coordinación y complementariedad de programas y proyectos.

Si este proyecto de ley se aprueba durante el segundo semestre de 2020, la educación superior y las políticas públicas para este nivel educativo contarán con directrices de mayor nitidez que antes.

\section{El programa sectorial de educación 2020-2024}

En México la planeación de las actividades gubernamentales es un derecho constitucional del pueblo de México, establecido en el Artículo 26 de nuestra Carta Magna, en él se establece que con base en un Sistema Nacional de Planeación Democrática los poderes ejecutivos nacional, estatal y municipal, deberán presentar a la representación popular su plan nacional de desarrollo (PND), su plan estatal de desarrollo y su plan municipal de desarrollo para el periodo de su mandato, así como los programas sectoriales (a cargo de una Secretaría de Gobierno) y especiales para actividades no sectorizadas. Es en este marco de obligatoriedad constitucional que se presentó el Programa Sectorial de Educación para el periodo 2020-2024 (Secretaría de Educación Pública, 2020). En este Programa el derecho a la educación está considerado dentro del Eje General 2. Política Social del PND 2019-2024, que articulará las acciones del gobierno federal en el ámbito educativo. Para atender las causas de fondo será necesario aplicar un doble enfoque. Por un lado, el sistema educativo ofrecerá educación relevante al entorno social, cultural, económico y geográfico del alumnado, así como inclusiva al reconocer sus necesidades, intereses, ritmos y talentos.

En este contexto, la política educativa de la presente administración se articulará en torno a seis prioridades, a saber: Educación para todas y todos, sin dejar a nadie atrás; Educación de excelencia para aprendizajes significativos; Académicos como agentes de la transformación educativa; Entornos educativos dignos y sana convivencia; Deporte para todas y todos; y Rectoría del Estado en la educación y consenso social.

Dentro de las estrategias prioritarias de este programa sectorial está garantizar la obligatoriedad y gratuidad de la educación media superior y superior como condición para asegurar el acceso de adolescentes y jóvenes al conocimiento, la cultura y el desarrollo integral, así como garantizar que los planes y programas de estudio sean pertinentes a los desafíos del siglo xxi y permitan a las niñas, niños, adolescentes y jóvenes adquirir las habilidades y conocimientos para su desarrollo integral. Para ello se plantean las estrategias de reorientar la formación continua del personal docente, directivo y de supervisión para el óptimo desempeño de sus funciones y la mejora continua del proceso de enseñanza-aprendizaje e impulsar el compromiso y la responsabilidad social para detonar un cambio de paradigma en la gestión del Sistema Educativo Nacional. Estas son las escasas estrategias dirigidas a transformar la gestión (estructuras, procesos, cultura organizacional, etc.) de la educación superior, siendo este un problema central para la mejora continua de la educación, puesto que si bien docentes y estudiantes han pasado por reformas y cambios en los modelos educativos, quienes son directivos y gestores, así como los modelos de gestión que aplican, no han tenido cambios en varias décadas. 
Estas estrategias se han traducido y seguramente seguirán traduciéndose en políticas públicas y formas de gestión para realizar las acciones propuestas y lograr los resultados previstos, pero necesitan complementarse y retomar el énfasis en estrategias de cambio para la gestión institucional y del subsistema de educación superior.

\section{Obligatoriedad y gratuidad de la educación superior}

Una innovación disruptiva en las políticas públicas para la educación superior se plasmó en la reciente Reforma Constitucional al establecer la obligatoriedad y gratuidad de todos los niveles educativos, incluyendo la educación superior. Esta reforma ha venido acompañada de políticas presupuestales para asegurar su cumplimiento, aunque no en los montos previstos por quienes dirigen las instituciones de educación superior públicas. La Asociación Nacional de Universidades e Instituciones Afines (ANUIES de la RM) consideró que:

En el Proyecto de Presupuesto no se incluye el fondo especial para dar cumplimiento al principio de obligatoriedad de la educación superior, al que se refieren la Fracción $X$ del Artículo Tercero Constitucional. Por lo anterior, en el marco de la gradualidad, será necesario asignar los recursos suficientes para contribuir al cumplimiento de los principios constitucionales de obligatoriedad y gratuidad, así como a la plurianualidad del financiamiento de la infraestructura (CUPIA, 2019).

Esto es, el Fondo para la Obligatoriedad de la Educación Superior. Las políticas de austeridad en el gasto y los impactos iniciales de la pandemia de la COVID-19 generaron una reducción de $4 \%$ del presupuesto federal, lo que aunado a un entorno internacional desfavorable a los precios del petróleo, fuente importante de recursos gubernamentales, han generado una crisis fiscal que limita el financiamiento público a la educación superior, que como otros sectores ya enfrentaba las políticas públicas de austeridad en el gasto gubernamental, además de la necesidad de dar cumplimiento a la Reforma Constitucional que impulsa la expansión de la oferta educativa con calidad en un proceso de mejora continua.

Así que:

Con el propósito de dar atención a lo dispuesto en la reforma al Artículo $3^{\circ}$. Constitucional, promulgada el 15 de mayo de 2019, cuyo mandato establece la obligatoriedad y gratuidad de la educación superior, la Cámara de Diputados del H. Congreso de la Unión aprobó una ampliación de 350 millones de pesos en el Presupuesto de Egresos de la Federación para el ejercicio 2020, asignados al Programa U079 Programa de Expansión de la Educación Media Superior y, para el tipo Superior (Subsecretaría de Educación Superior, 2020).

Cantidad inferior a la prevista y solicitada por la Asociación Nacional de Universidades e Instituciones de Educación Superior (ANUIES de la RM), asociación que representa a las principales instituciones en el nivel nacio- 
nal, por lo cual este recurso solo fue asignado a las normales, tecnológicas y universidades interculturales, así como algunas universidades autónomas de los Estados con mayor rezago educativo en la matrícula de educación superior.

Por otro lado, la educación superior privada en México atiende un poco más de $30 \%$ de la matrícula de este nivel educativo y han previsto una reducción de su matrícula lo que llevará a una disminución de sus ingresos de alrededor de $25 \%$, debido tanto a la reducción de los ingresos familiares ante el paro parcial y disminución de la actividad económica debido a la COVID-19, a la pérdida de ingresos de estudiantes que trabajan para mantenerse y pagar sus estudios, así como a la ampliación de la matrícula que han realizado y seguirán realizando las universidades públicas, lo que según algunos cálculos implicará que $40 \%$ de estas instituciones cerrará sus puertas, en especial las llamadas universidades de absorción de demanda, aquellas que atienden la demanda de estudiantes no aceptados en las universidades públicas, y que cobran colegiaturas bajas, comparadas con las de instituciones de educación superior más grandes y más conocidas.

Ante esta situación se han dado dos fenómenos, el aumento en la demanda de servicios educativos para las instituciones de educación superior pública, ya que muchos de los estudiantes que dejarán las instituciones privadas han buscado cupo en las públicas, y la búsqueda de apoyos gubernamentales para las instituciones privadas, las cuales están promoviendo un acuerdo con los gobiernos de algunas de las entidades federativas (como es el caso del estado de Jalisco), para crear una bolsa gubernamental para subsanar sus gasto de operación.

Pero el abandono escolar no se manifiesta únicamente en las instituciones privadas, es un fenómeno que ya se presenta en todo el subsector de educación superior y que según la Secretaría de Educación Pública será para este año de 305,000 estudiantes, $8 \%$ de la matrícula total actual. Por ello el 8 de julio de 2020 se realizó la asignación de recursos a Instituciones Públicas de Educación Superior en la vertiente A del Programa U079, correspondiente a apoyos presupuestales a proyectos que incrementarán la matrícula de educación superior en Universidades Públicas e Institutos Tecnológicos, consistentes en mejoramiento, ampliación o creación de infraestructura y equipamiento, creación y consolidación de nuevos programas de estudio, fortalecimiento de capacidades institucionales y habilidades estudiantiles técnicas y tecnológicas básicamente para entidades federativas con coberturas más bajas que el promedio nacional (Oaxaca, Chiapas, Guerrero, Tlaxcala, Quintana Roo, San Luis Potosí y Michoacán) con excepción del Estado de México, o con necesidades específicas para ampliar la matrícula en otras entidades federativas. De igual forma se realizó la asignación de recursos a Instituciones Públicas de Educación Superior en la vertiente $B$ del Programa U079, correspondiente a apoyo a la gratuidad de los servicios de educación superior para ampliar la matrícula en universidades interculturales indígenas y en Escuelas Normales, Institutos y Centros de formación para el magisterio en entidades federativas como Zacatecas, Tlaxcala, Tabasco, Sonora, Michoacán, Hidalgo, Guanajuato, Durango, Coahuila, Ciudad de México, Campeche y Baja California Sur. 


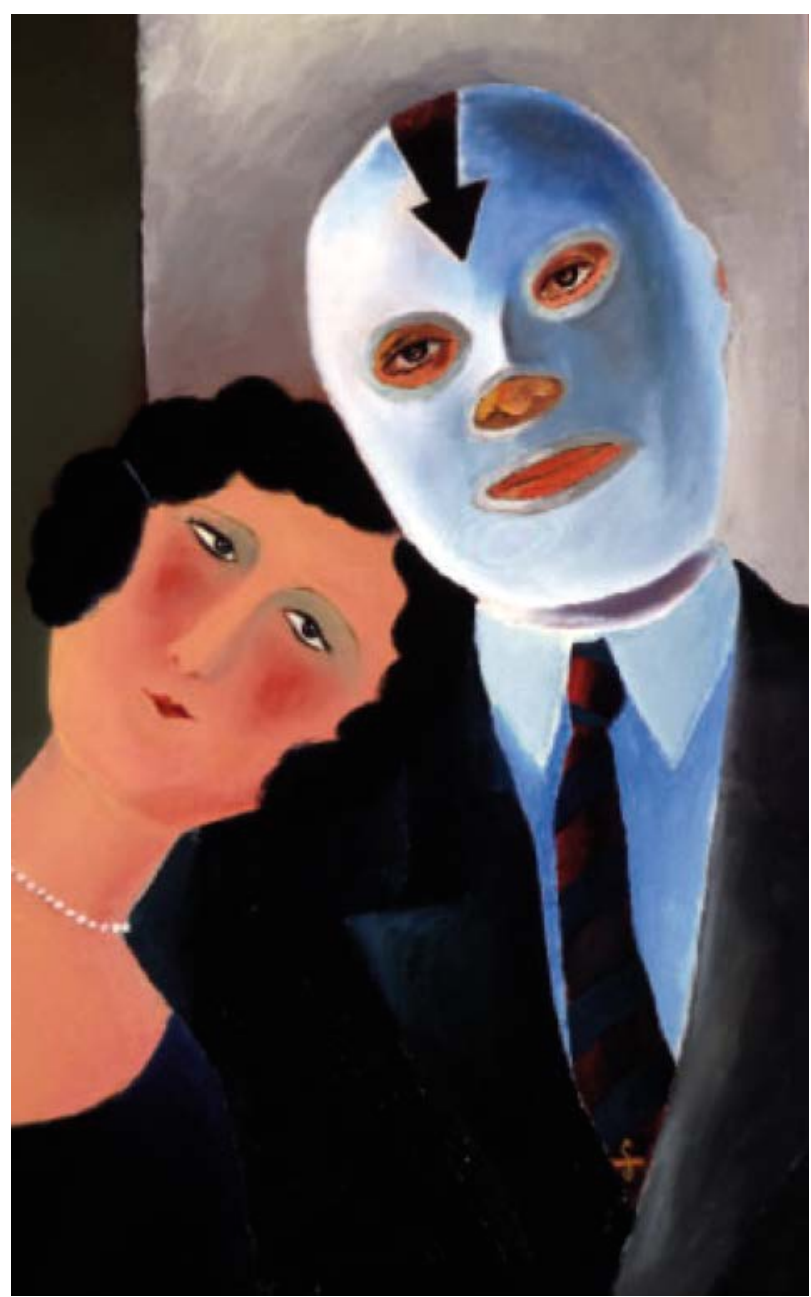

Los esposos shadow (detalle). Óleo sobre tela, 70 x 80 cm. 1986.
La política presupuestal ha buscado impactos en el logro de las metas para la educación superior planteadas en el Programa Sectorial de Educación, y está dando respuestas puntuales y dirigidas a las entidades y a las instituciones más desfavorecidas en el contexto social y económico actual. Sin embargo, estas respuestas aún no tienen un eje vertebrador integral que dé nuevas directrices a la asignación presupuestal derivada de la política de financiamiento público a la educación superior, en sus directrices sigue prevaleciendo la continuidad de las políticas anteriores, lo cual es explicable al tener apenas un poco más de un año en el gobierno la actual administración, pero hay enormes expectativas para que esto cambie favorablemente a un nivel educativo.

Con base en la política pública de austeridad en el gasto del gobierno federal se expidió un decreto para extinguir fideicomisos públicos y otras figuras jurídicas afines (Secretaría de Gobernación, 2020) que no tuvieran estructura orgánica, que carecieran de mandatos y análogos, ya que algunos de estos fideicomisos fueron creados con fines que respondían a intereses específicos y algunos de ellos se utilizaron para desviar recursos públicos que implicaban un gasto equivalente a $1 \%$ del Producto Interno Bruto (PIB) del país enviando todos los recursos al financiamiento de Proyectos Nacionales de Investigación e Incidencia (PRONAII) que se encuentran alineados a los programas estratégicos. Esta 
medida obliga a los Centros Públicos de Investigación del Consejo Nacional de Ciencia y Tecnología (CONACYT) que sus proyectos se encuentren alineados directamente a los Programas Nacionales Estratégicos (PRONACES) del CONACYT, cuya función es organizar los esfuerzos de investigación en torno a problemáticas nacionales concretas, que van del planteamiento del problema o el reto a la articulación de capacidades científico-técnicas y de colaboración con actores sociales, sector público o privado para ello. Entre los que se encuentran:

1. Salud.

2. Soberanía alimentaria, producción de alimentos santos y agroecología campesina.

3. Desarrollo urbano-industrial, desechos sólidos y toxicidades.

4. Prevención de riesgos y desastres.

5. Conocimiento y gestión de cuencas del ciclo socio-natural del agua, para el bien común y la justicia socio-ambiental.

6. Cambio climático y calidad del aire.

7. Transición energética y cambio climático, con modelos tecnológicos de bajo consumo de energía aplicados a la ciudad y el campo.

8. Movilidad humana.

9. Vivienda sustentable y pertinente, cultural y ambientalmente.

10. Ciudades sustentables.

11. Sistemas socioambientales y sustentabilidad: conservación de ecosistemas terrestres, costeros y marinos con manejo y producción sustentables.

12. Violencias estructurales.

13. Educación para la inclusión y la autonomía.

14. Memoria histórica y procesos bioculturales de México.

Por tanto, los proyectos que no se encuentren en este marco no podrán ser apoyados financieramente.

Otras medidas de austeridad del gobierno federal del nuevo régimen fue determinar que ningún funcionario público podría ganar más que el Presidente de la República, medida que ha sido difícil aplicar en las universidades y otras instancias del gobierno que gozan de autonomía, de ahí la necesidad, como se planteaban antes, de una Ley de Autonomía Universitaria. Esta medida busca acabar con lo que se conoce como "la burocracia dorada" y dedicar recursos a aumentar la matrícula hasta lograr la cobertura universal

\section{Continuidad en algunas políticas públicas para la educación superior}

Entre las continuidades que presentan las políticas públicas del actual régimen de gobierno federal está la permanencia del Programa para el Desarrollo Profesional Docente, para el tipo Superior (SES, 2020) donde se mantienen los tipos de apoyo dirigidos a las Instituciones de Educación Superior Públicas que participen en el Programa y que tienen un alto impacto en las Instituciones de Educación Superior Privadas (IESP), ya que muchos de los apoyos e ingresos extra del profesorado dependen de que sus instituciones 
participen. Este Programa da apoyos para que el profesorado de tiempo completo:

Realice estudios de posgrado y realice sus tesis; dando reconocimiento a perfil deseable y apoyo a profesores que cumplen, con eficacia y equilibrio en sus funciones de profesor de tiempo completo, como atender la generación y aplicación del conocimiento, ejercer la docencia y participar en actividades de tutorías y gestión académica, así como dotar de los implementos básicos para el trabajo académico a los profesores reconocidos con el perfil; apoyo a la incorporación de nuevos profesores de tiempo completo (PTC) y apoyo a la reincorporación de exbecarios (SES,2020).

Del Programa y apoyos para la conformación de Cuerpos Académicos (CA), que lo forman:

Profesores-investigadores que comparten una o más líneas de estudio, cuyos objetivos y metas están destinados a la generación y/o aplicación de nuevos conocimientos. Además, por el alto grado de especialización que alcanzan en conjunto al ejercer la docencia, logrando una educación de buena calidad (SES,2020).

Hasta el presente ejercicio fiscal y en los planteamientos realizados por las autoridades de este nivel educativo, esta política de apoyos extraordinarios y diferenciados, sometidos a concurso para las IESP no parecen tener cambios.

Sin embargo, se han anunciado cambios importantes en las reglas del CONACYT:

Para calificar al Programa Nacional de Posgrados de Calidad (PNPC), se introdujeron tres nuevos concursos, uno tiene que ver con el acento en la interculturalidad y con la vinculación de esos posgrados con la sociedad circundante, y el otro trata el tema de lo étnico en el centro y las condiciones de vinculación entre las instituciones y sus comunidades directas (Concheiro, 2020).

Así como en los criterios para el sNi, donde a los ya existentes se incluyeron criterios de impacto social semejantes a los del posgrado como elementos importantes para medir la productividad, así como el impulso a los Clubes por la Paz, en el marco de una cultura de paz y de no violencia en las IEs, llevados a cargo de los estudiantes y de la Institución. Un cambio importante en las políticas públicas actuales es tener como uno de sus marcos referenciales a la economía social y solidaria y no solo a la economía de mercado (Concheiro, 2020), lo que perfila una nueva generación de políticas para la educación superior. 


\section{Aparece un cisne negro. Las políticas y acciones gubernamentales e institucionales ante la pandemia de la COVID-19}

La aparición de la pandemia provocada por la llamada CoviD-19, resultó un verdadero cisne negro (Taleb, 2007), un evento tan inesperado como de consecuencias catastróficas en todos los sectores de la economía, la sociedad y también en la educación, ya que las instituciones de todos los niveles educativos cerraron sus puertas ante la emergencia sanitaria de COVID-19 y de forma urgente implementaron estrategias y acciones para atender la continuidad del ciclo educativo apoyadas en las tecnologías de la Información y la Comunicación (TIC). Para conocer las acciones inmediatas realizadas por las instituciones de educación superior se realizó una encuesta organizada por la Dirección General de Educación Superior Universitaria (DGESU) de la Subsecretaría de Educación Superior (SES) en coordinación estrecha con la ANUIES de la RM.

La encuesta aplicada a las IES arroja un alto porcentaje de continuidad de las actividades académicas durante la emergencia sanitaria. $100 \%$ de las instituciones encuestadas (138) reportaron que, durante la emergencia sanitaria continuaron con sus actividades académicas transitando a la modalidad a distancia. En promedio las instituciones reportaron que el porcentaje de alumnos atendidos en la modalidad a distancia fue de $92 \%$ al 8 de mayo y de $71 \%$ al 8 de junio (Secretaría de Educación Superior, 2020b).

Esto significa que ha disminuido el porcentaje de estudiantes atendidos en las instituciones encuestadas:

Las instituciones reportaron que en promedio $69 \%$ de sus docentes están preparados para continuar sus labores académica ahora en la modalidad no escolarizada (...) y que los principales obstáculos o carencias informáticas que enfrentan son, falta total o insuficiencia de infraestructura tecnológica; saturación de la red para el uso de las plataformas o mala calidad de la señal de Internet; carencia de equipo de cómputo o internet/equipo inservible para la realización de las actividades; desconocimiento de alumnos o docentes en el uso y manejo de las plataformas utilizadas para educación a distancia; poca disponibilidad de material y herramientas digitales (Secretaría de Educación Superior, 2020b).

No existe un censo de estudiantes ni de docentes que brinde información sobre las condiciones para el trabajo a distancia, ni sobre la disponibilidad de equipo y de conectividad. Al inicio de la pandemia algunas instituciones como la Universidad de Guadalajara (Gómez, et al.) tomaron el liderazgo en recabar esta información y en diseñar acciones para compensar estas carencias, como la donación o préstamo de equipo de cómputo, acceso gratuito a Internet, etc., pues como lo señala la encuesta antes mencionada (SES-ANUIES), 26\% del estudiantado no cuenta con computadora o tablet y no tiene acceso a internet de manera constante en casa. 
La encuesta señala que con el fin de continuar con las labores docentes, las instituciones mencionan realizar las siguientes actividades con los docentes y alumnos: Asistencia técnica para el uso de plataformas, capacitación a docentes y alumnos, implementación de nuevos procesos para el seguimiento y la evaluación de las actividades, elaboración y facilitación de nuevos materiales académicos y bibliotecas digitales, préstamo de equipo de cómputo e implementación de trámites administrativos y de procesos de titulación en la modalidad a distancia.

Pero centrar la atención solo en la tecnología no da una perspectiva completa de la problemática a la que ha enfrentado esta pandemia, ni de las políticas públicas para enfrentarla con éxito en la educación superior, se debe considerar tanto en las políticas públicas como en las acciones institucionales, que también un porcentaje similar de estudiantes no cuentan con condiciones dentro del hogar para recibir la educación en línea, que las condiciones de su vivienda no les permiten contar con un espacio exclusivo para ello, y que el equipo de cómputo existente es compartido entre los miembros de la familia que trabajan en casa (el home office) y otros miembros que también estudian, además de la inexistencia o las deficiencias en el suministro de energía eléctrica por lo deteriorado de las conexiones, así como el abandono escolar que se enfrentará.

La Secretaría de Educación Pública (SEP) ha planteado recientemente que la pandemia originada por la COVID-19 ha agrandado el reto de la equidad educativa, que puede llevar al sistema educativo a consecuencias muy graves y de impactos negativos imprevistos si no se diseñan y aplican políticas públicas imaginativas, innovadoras y disruptivas, pero sobre todo, sustentadas en la amplia colaboración solidaria de todas las instituciones educativas. Esta cooperación solidaria entre gobierno e IESP, se ha dado en varias "vertientes de acción de las IES ante la COVID-19: a) colaboración en la Jornada Nacional de Sana Distancia", apoyo a las autoridades sanitarias y auxilio a la población; b) Continuidad de la docencia, investigación y difusión de la cultura con apoyo de las tecnologías de la información y la comunicación a distancias (TICAD), y c) vinculación con los sectores productivos para detonar las economías locales y regionales" (Secretaría de Educación Superior 2020b), pero también las IESP han impulsado acciones para proveer de despensas a los estudiantes más necesitados y a sus familias, poner las instalaciones al servicio de la toma de muestras, atención a pacientes diagnosticados con COVID-19, e incluso proporcionar espacio gratuito para la reclusión individual o familiar (como lo hizo la Universidad de Guadalajara) de quienes estén en este caso y no tengan condiciones para hacerlo en casa.

Resulta apremiante acelerar el proceso de cambio y fortalecer la construcción de ecosistemas de aprendizaje en general, con el liderazgo y corresponsabilidad del subsistema de educación su- 
perior, que hasta ahora ha tenido escasos vínculos con la educación básica y obligatoria y que, como lo señala el Programa Sectorial de Educación, tiene el compromiso de vertebrarse con todo el sistema educativo.

Respecto a las actitudes para el uso de las TIC, la percepción de su utilidad y sus determinantes, Olaskoaga, Rodríguez y Marúm (2018, p. 159) plantean que:

Una amplia mayoría de los encuestados (se declara de acuerdo o muy de acuerdo con que la introducción de las TI mejora la actividad docente del profesor(a) $(85,1 \%)$ y contribuye a fomentar la innovación educativa $(87,5 \%)$. Son datos que confirman la buena acogida que los docentes han otorgado a las TI en su trabajo, y que obligan a abandonar el estereotipo que describe al profesorado de la ES como un grupo conservador que se opone a cualquier novedad en su trabajo.

Por lo que el profesorado no solo de la educación superior, sino de todos los niveles educativos ha demostrado su flexibilidad, compromiso y responsabilidad para transitar a la virtualidad, a pesar de todos los retos y dificultades suscitados.

\section{Referencias}

Brunner, José Joaquín y Daniel Andrés Miranda (Editores) (2016). Educación Superior en Iberoamérica. Informe 2016. Chile, Centro Interuniversitario de Desarrollo (CINDA), 2016.

Concheiro, Luciano (2020). Conferencia "La autonomía universitaria en el nuevo modelo de educación pública superior”. Puebla, México, Benemérita Universidad Autónoma de Puebla, agosto.

Consejo de Universidades Públicas e Instituciones Afines (2019). Presupuesto de Egresos de la Federación 2020. Solicitud de ampliación al presupuesto para la educación superior. México, ANUIES, CUPIA.

Gobierno de México (2020). El futuro de la educación superior en México ante la COVID-19. México, Secretaría de Educación Pública, Subsecretaría de Educación Superior, Dirección General de Educación Superior Universitaria.

Gómez Estrada, Mateo, María Celeste Godoy Castro y Yolanda Quintero Maciel (próxima publicación). Benchmarking de Acciones de Tres Universidades Estatales Mexicanas ante la Pandemia Covid-19. Universidad de Guadalajara, Universidad Autónoma de Baja California y Universidad Autónoma de Tamaulipas.

Marúm Espinosa, Elia (Especialista Principal) (2016). "Educación Superior en Iberoamérica. Informe Nacional: México.” En: Brunner, José Joaquín y Daniel Andrés Miranda (Editores) (2016). Educación Superior en Iberoamérica. Informe 2016. Chile, Centro Interuniversitario de Desarrollo (CINDA).

Marúm Espinosa, Elia. (octubre 2012). "El Posgrado en México y su Responsabilidad Social Ampliada ante la Sustentabilidad del Desarrollo Nacional". Revista Argentina de Educación Superior, año 4, núm. 5.

Marúm Espinosa, Elia (octubre, diciembre 2019). "Estado de la situación educativa en México y propuestas de mejora a raíz de la Reforma Educativa”. Revista Mexicana de Investigación Educativa, vol. 24, núm. 83, México

Olaskoaga Larrauri, Jon, Carmen Enedina Rodríguez Armenta y Elia Marúm Espinosa (20 junio 2018). "Nociones de calidad educativa y actitudes de los docentes 
hacia las tecnologías de la información”. Revista Internacional de Organizaciones, núm. 20, pp. 147-168.

Secretaría de Educación Pública (junio 7 2020). Programa Sectorial de Educación 2020-2024. Diario Oficial de la Federación (DOF): 06/07/2020.

Secretaría de Gobernación (2020). Decreto por el que se ordena la extinción o terminación de los fideicomisos públicos, mandatos públicos y análogos. 2 de abril de 2020, Diario Oficial de la Federación. DOF: 02/04/2020. Recuperado de: https:// www.dof.gob.mx/nota_detalle.php?codigo $=5591085 \&$ fecha $=02 / 04 / 2020$

Secretaría de Gobernación (2020a). Decreto por el que se establecen las medidas de austeridad que deberán observar las dependencias y entidades de la Administración Pública Federal bajo los criterios que en el mismo se indican. DOF: 23/04/2020. Recuperado de: http://dof.gob.mx/nota_detalle.php?codigo $=5592205 \&$ fecha $=23 / 04 / 2020$

Subsecretaría de Educación Superior (octubre 2019). Anteproyecto de Ley General de Educación Superior. Recuperado de: https://consulta-ley-educacion-superior. mx/wp-content/uploads/2019/11/ANTEPROYECTO-LGES-Octubre-2019.pdf

Secretaría de Educación Superior (2020). Políticas de Apoyo a la Educación Superior Subsecretaría de Educación Superior, Página Oficial https://educacionsuperior. sep.gob.mx

Subsecretaría de Educación Superior (2020a). Programa de Expansión de la Educación Media Superior y Superior, para el tipo superior U079 Ejercicio 2020. México, SEP. Recuperado de: https://educacionsuperior.sep.gob.mx/pdfs/2020/ expansionU079.pdf

Subsecretaría de Educación Superior (2020b). El futuro de la Educación Superior en México ante la COVID-19. México, Dirección General de Educación Superior Universitaria, SEP.

Taleb, Nassim Nicholas (2007). The Black Swan: The Impact of the Highly Improbable. New York, Random House. 